\title{
Volumetric laser endomicroscopy and optical coherence tomography in Barrett's esophagus: a systematic review and meta-analysis
}

\section{(ㄷ)(i)}

\author{
Authors \\ Maria Auxiliadora Chóez Rodriguez ${ }^{1}$, Diogo Turiani Hourneaux de Moura2๑, Igor Braga Ribeiro1๑, Wanderley \\ Marques Bernardo $^{1 \odot}$, Flavio Hiroshi Ananias Morita ${ }^{1 \odot}$, Sergio Barbosa Marques ${ }^{1 \odot}$, Paulo Sakai ${ }^{1 \odot}$, Eduardo \\ Guimarães Hourneaux de Moura1®
}

Institutions

1 Department of Endoscopy, Hospital das Clínicas, University of São Paulo School of Medicine, São Paulo, Brazil

2 Division of Gastroenterology, Hepatology and Endoscopy, Brigham and Women's Hospital, Harvard Medical School, Boston, Massachusetts, United States

submitted 23.11.2019

accepted after revision 29.5.2019

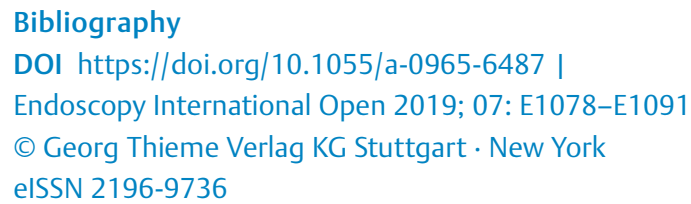

\section{Corresponding author}

Igor Braga Ribeiro, MD, Academic Fellow, Surgeon, Gastrointestinal Endoscopy Unit, Hospital das Clínicas da Faculdade de Medicina da Universidade de São Paulo, Av. Dr. Enéas de Carvalho Aguiar 255, Instituto Central, Prédio dos Ambulatórios, Pinheiros, São Paulo 05403-000, Brazil Fax: +551130697579 igorbraga1@gmail.com

\section{ABSTRACT}

Background and study aims Endoscopic imaging of Barrett's esophagus (BE) with advanced technologies, such as optical coherence tomography (OCT) and volumetric laser endomicroscopy (VLE), allows targeted biopsies and may reduce the number of random biopsies to detect esophageal neoplasia in the early stages during endoscopic BE surveillance. The aim of this study was to evaluate the accuracy of OCT and VLE in diagnosis of intestinal metaplasia, dysplasia, and high-grade dysplasia (HGD), and intramucosal carcinoma (IMC) in BE.

Patients and methods In this systematic review and meta-analysis, the primary outcome measure was diagnostic accuracy of OCT and VLE, in comparison with the gold standard. In the meta-analysis, we calculated sensitivity, specificity, positive likelihood ratio $(L R+)$, negative likelihood ratio (LR-), and diagnostic odds ratio (DOR) for both methods. We performed analyses by patient and by lesion. Results We evaluated 14 studies involving a collective total of 721 patients and 1565 lesions. In the analysis by lesion, VLE showed a pooled sensitivity, specificity, LR+, LR-, DOR, and SROC AUC of $85 \%, 73 \%, 3.2,0.21,15.0$, and 0.87 , respectively, for detection of $\mathrm{HGD/IMC}$. In the analysis by lesion for detection of HGD/EAC, OCT showed a pooled sensitivity, specificity, LR+, LR-, DOR, and summary receiver operating characteristic area under the curve of $89 \%, 91 \%$, $9.6,0.12,81.0$, and 0.95 , respectively. The accuracy of OCT in identifying intestinal metaplasia showed a pooled sensitivity, specificity, LR+, LR-, and DOR of $92 \%, 81 \%, 5.06$, 0.091 , and 55.58 , respectively.

Conclusion OCT- and VLE-guided targeted biopsies could improve detection of dysplasia and neoplasia. Further studies could determine whether the use of such biopsies might replace the current protocol.

\section{Introduction}

In the United States, incidence of esophageal squamous cell carcinoma has decreased over the last few decades, whereas that of esophageal adenocarcinoma (EAC) has increased [1]. Endoscopic surveillance aims to alter the natural history of the disease by identifying esophageal neoplasia in its early stages, thus allowing curative endoscopic therapy to be instituted [2].

In recent years, advanced imaging enhancement techniques such as confocal laser endomicroscopy (CLE) and chromoscopy with narrow-band imaging (NBI) have been developed to improve the detection of dysplasia and adenocarcinoma in Barrett's esophagus (BE) [3]. However, current guidelines still ad- 
vocate use of the classic endoscopic surveillance protocol [2, 3], in which multiple biopsies are necessary, especially in cases of long $B E$, which requires greater technical expertise and time. Therefore, endoscopists rarely adhere to that guideline in such cases, leading to a considerable number of cases going underdiagnosed [3].

In studies involving small patient samples, it has been demonstrated that image enhancement techniques increase the detection of dysplasia in $\mathrm{BE}$, although use of such techniques is restricted to tertiary care centers, thus limiting generalization of the results to other centers [4].

One such technique is CLE, which allows adequate evaluation and visualization of short BE and specific areas that are suspicious. However, using CLE to evaluate a long BE requires longer endoscopy times, making it exhaustive.

Optical coherence tomography (OCT) and volumetric laser endomicroscopy (VLE) are new technologies that use infrared light, allowing acquisition of high-resolution microscopic images, in real time, without need for contrast. OCT is an optical imaging technique consisting of an infrared light catheter, which obtains cross-sectional images of tissues evaluated in high resolution, analogous to ultrasound but using infrared light rather than acoustic energy. Transverse images obtained have a resolution of $10 \mu \mathrm{m}$, which is 10 times better than that of high-frequency ultrasound [5]. The evaluation consists in introducing the catheter through the working channel of a conventional endoscope and positioning the catheter over the specific area of interest to be analyzed.

The images are acquired by linear longitudinal scanning of the length and depth, the scan dimensions varying according to the catheter used. Sequential image frames are continuously obtained and updated at a rate of four frames per second (fps), as well as being numbered consecutively during acquisition for reference and subsequent data analysis [5,6]. After acquisition of the images, the catheter is removed and biopsy forceps can immediately be inserted through the working channel in order to biopsy any suspicious area of mucosa observed during the procedure. OCT devices have evolved from their first incarnation to the current catheters, presenting improvements not only in axial and transverse resolution, allowing evaluation of microvascular characteristics such as OCT angiography (OCTA) improving detection of low- and high-grade dysplasia (LGD and HGD, respectively), but also in speed of image acquisition, as well as in linear-scanning diameters, through incorporation of micromotor catheters that allow upper axial scans with a velocity 100 times greater (400 fps) [7]. Axial and transverse resolution of the catheters used in the studies ranged from $10 \mu \mathrm{m} \times$ $25 \mu \mathrm{m}$ [6] to $8 \mu \mathrm{m} \times 20 \mu \mathrm{m}$ [7] and $5 \mu \mathrm{m} \times 5 \mu \mathrm{m}$ [8,9], the last with five times better resolution, available in ultra-high resolution OCT (UHR-OCT) and three-dimensional OCT (3 D OCT) [9]. The linear-scanning diameters (length $\times$ depth) are $3 \mathrm{~mm} \times 2.5$ $\mathrm{mm}$ [8], $5.5 \mathrm{~mm} \times 2.5 \mathrm{~mm}$ [6], $10 \mathrm{~mm} \times 16 \mathrm{~mm}$ [7], and $8 \mathrm{~mm} \times$ $20 \mathrm{~mm}$ [9], which together with the sequential acquisition frame rate - $4 \mathrm{fps}$ [6], $60 \mathrm{fps}$ [9], or $400 \mathrm{fps}$ [7] - depends on the OCT catheter used. External diameters of the catheters vary from $1.8 \mathrm{~mm}$ to $2.0 \mathrm{~mm}$ and $2.5 \mathrm{~mm}$.
VLE is second-generation, advanced OCT that uses near-infrared light and provides high-resolution cross-sectional images in real time, the technology involving balloon-centered imaging probes, a console and monitor [10]. The probe is introduced through the working channel of the therapeutic endoscope and centralized by a balloon, available in diameters of $14 \mathrm{~mm}, 17 \mathrm{~mm}$, and $20 \mathrm{~mm}$, with a length of $6 \mathrm{~cm}$. Imaging is performed by automatic helical pullback of the probe from the distal to the proximal end of the balloon over a 90-second period. VLE images have an axial resolution of $7 \mu \mathrm{m}$, have a transverse resolution of 30 to $40 \mu \mathrm{m}$, and can reach a depth of up to $3 \mathrm{~mm}$, allowing detailed visualization of the esophageal mucosa and submucosa. A total of 1200 cross-sectional images are acquired over a 6-cm VLE scan [10]. It is an interesting option because it allows larger BE segments to be evaluated in a shorter time [10]. Reconstruction of the images is done in the console allowing real-time diagnosis of esophageal mucosa abnormalities, as well as guiding endoscopic treatment. VLE with laser marking (VLEL) has become available, and it is possible to apply VLE-guided superficial cauterization marks on the esophageal mucosa, without the need to change devices. Those temporary marks allow the endoscopist to refer directly to the tissue for subsequent direct histological sampling or to delineate a lesion for subsequent resection [11].

In a systematic review evaluating accuracy of OCT in the identification of dysplasia and early-stage cancer, Kohli et al. [12] reported that the technique had a sensitivity of $68 \%$ to $83 \%$ and a specificity of $75 \%$ to $82 \%$. To our knowledge, there have been no previous systematic reviews evaluating accuracy of VLE in BE. There have also few studies assessing accuracy of OCT and VLE. This is the first systematic review that evaluates accuracy of VLE in identification of dysplasia and neoplasia in BE.

\section{Methods}

\section{Study protocol and registration}

The study protocol, including the search strategies, inclusion criteria, and methods of statistical analysis, was previously established and registered in the PROSPERO database (http:// www.crd.york.ac.uk/prospero) under no. CRD42018089362.

\section{Eligibility criteria}

We selected prospective and retrospective observational studies that employed OCT and VLE in surveillance of patients with $\mathrm{BE}$ and provided sufficient data to calculate sensitivity, specificity, positive likelihood ratio $(L R+)$, negative likelihood ratio $(\mathrm{LR}-)$, diagnostic odds ratio (DOR), and area under the summary receiver operating characteristic curve (SROC AUC), either by patient or by lesion, regardless of the primary outcome defined by the authors of the studies. Studies evaluating squamous cell carcinoma or other types of esophageal neoplasms were excluded. 


\section{Types of subjects}

Only studies involving individuals with a histological diagnosis of BE and under endoscopic surveillance were included. We imposed no restrictions regarding the characteristics of the subjects (gender, age, risk factors, comorbidities, time since BE diagnosis, or surveillance after endoscopic eradication for dysplasia or early-stage cancer)

\section{Diagnostic methods}

We included studies on use of OCT or VLE for identification of intestinal metaplasia (IM), LGD, HGD, and intramucosal carcinoma (IMC) in BE. The gold standard for comparison of diagnostic methods was histopathological analysis of specimens obtained by biopsy, endoscopic mucosal resection, or endoscopic submucosal dissection of suspicious and apparently normal areas.

\section{Types of outcome measures}

We selected studies in which the primary outcome measure was diagnostic accuracy of OCT and VLE in identifying of IM and LGD when feasible in the studies, as well as the identification of HGD and IMC in BE.

\section{Search strategies}

We performed a search of the literature, up through mid-January 2019, via the following indices: Medline (PubMed); Excerpta Medica; Cochrane Central Register of Controlled Trials (CENTRAL); Literatura Latinoamericana y del Caribe en Ciencias de la Salud (LILACS, Latin-American and Caribbean Health Sciences Literature); and Scopus. We also conducted hand searches of the bibliographies of the studies selected. The search strategies varied by database:

Medline (PubMed) - (esophagus OR esophageal) AND (neoplasms OR cancer OR adenocarcinoma OR dysplasia OR dysplastic OR Barrett OR metaplasia) AND (narrow band imaging OR optical imaging OR NBI OR chromoendoscopy OR chromoscopy OR indigo carmine OR acetic acid OR methylene blue OR virtual imaging OR FICE OR flexible spectral imaging color enhancement OR i-scan OR BLI OR blue laser imaging OR high definition OR confocal laser endomicroscopy OR AFI OR autofluorescence imaging OR volumetric laser endomicroscopy OR VLE OR endoscopic optical coherence tomography OR OCT OR endoscopy OR endoscopic).

Excerpta Medica - Barrett esophagus AND volumetric laser endomicroscopy AND optical coherence tomography.

CENTRAL and LILACS - Barrett esophagus AND optical coherence tomograph.

Scopus - Barrett esophagus AND optical coherence tomography AND volumetric laser endomicroscopy.

\section{Study selection}

Two independent reviewers evaluated the titles and abstracts of the articles initially identified. Disagreements were resolved by consensus, in consultation with the other authors.

Because OCT and VLE are new technologies that have not been widely studied, we did not exclude studies that interpret- ed image datasets or used offline evaluation. We also included conference abstracts, as long as they allowed extraction of all data and those data were clearly presented, given that our objective was to generate a meta-analysis and not just a systematic review. When more than one article reported the same study, we selected the article that provided the most information. The analysis included four types of results: detection of IM; detection of dysplasia in general (LGD, HGD, or EAC), when it was possible to perform those types of analyses; identification of HGD; and identification of IMC.

\section{Data collection}

Data were collected in the form of absolute values that were provided directly or were inferred in the text. These data were extracted into $2 \times 2$ tables including true-positive, false-positive, true-negative, and false-negative results, to perform the different types of analyses and subgroup analyses, either by patient or by lesion. When the studies provided sufficient data to perform the various types of subgroup analyses, the data were included according to the positivity criterion for the analyzed group, independent of the primary outcome of the study. Otherwise, subgroup analysis was performed if feasible. When data were inconclusive or missing, we contacted the authors. If a study included multiple outcomes from multiple evaluators, the result from the best evaluator was used in the calculations; when the best evaluator was not identified, the mean of the results was used. The entire process was concluded by two of the authors, working independently, and was reviewed by all of the authors. Disagreements were resolved by consensus.

\section{Criteria for positivity}

Criteria applied in order to categorize a result as positive were established by the authors of each study, and we honored those categories for extraction of the data, provided that they were suitable for analysis. When interpreting the images obtained with VLE in the studies selected, we used diagnostic criteria for positivity that were based on diagnostic algorithms, scores, or simply the mention of suspicious findings, in order to determine the type of diagnosis in non-neoplastic lesions (indefinite for dysplasia or LGD) or neoplasia (HGD or IMC). Unfortunately, those diagnostic criteria were not standardized across the studies. A positive result was defined according to the criterion proposed by the author(s). Although the scores are not standardized, there is considerable overlap in the criteria identified, because they are based on the description of the suspicious findings found in the evaluations employing OCT and VLE.

Among the OCT scores is the OCT dysplasia index, and we found that, when a cut-off score $\geq 2$ was applied, the index had a sensitivity of $83.3 \%$ and a specificity of $75.0 \%$ for diagnosis of IMC and HGD. In the OCT dysplasia index, the main findings for HGD/IMC positivity are surface maturation (surface OCT signal stronger than subsurface $=2$ ) and gland architecture (moderate/severe irregularity, highly asymmetric dilated glands, or debris within the gland lumen $=2$ ). For diagnosis of IM, the suspicious OCT findings are as follows: absence of the layered structure of normal squamous epithelium and of the vertical "pit and crypt" morphology of normal gastric mucosa; disorga- 
nized architecture with irregular mucosal surface; and submucosal glands of low reflectance below the epithelial surface or invaginations through the epithelium.

In the studies employing VLE, the following are the main findings for HGD/IMC positivity: effacement of the mucosal layer, defined as layering in $<50 \%$ of the scan; signal intensity distribution (surface signal > subsurface signal); and gland architecture ( $>5$ irregular glands). Details of the scores and scoring are shown in $>$ Fig. 1 .

\section{Risk of bias in individual studies}

Two independent reviewers assessed quality of the studies included in the meta-analysis on the basis of predefined criteria and discussions involving the remaining authors. To facilitate that process, we used the Quality Assessment of Diagnostic Accuracy Studies, version 2 (QUADAS-2) [13], the criteria for which were used to analyze risk of bias and applicability in the patient selection process; how the OCT and VLE were conducted and interpreted; the way in which the lesions were classified in the histopathological evaluation; and the clinical significance of the findings.

Cross-sectional studies with adequate homogeneity between the groups were evaluated with the technologies under study. Risk of bias in patient selection was considered unknown when the patient selection process was not clearly defined. Applicability of patient selection was considered low when the included patients were undergoing BE surveillance or follow-up after endoscopic treatment for dysplasia or IMC.

We evaluated whether lesion classifications were standardized and whether an appropriate criterion for positivity was used; if not, risk of bias was considered high. For the gold standard (biopsy), blinding the pathologist to the endoscopic findings effectively reduced risk of bias and increased applicability. For studies in which LGD was considered a true-positive result and there were sufficient data to distinguish LGD from HGD and adenocarcinoma, LGD findings were reclassified as true-positive results and included in the subgroup analysis. If the final outcome was not assessed in all patients included in the studies, the risk of bias was considered high.

\section{Statistical analysis}

For the meta-analysis, we used STATA IC/64 software, version 13.1 (Stata Corp., College Station, Texas, United States), with the MIDAS and METANDI modules, and the Statistical Analysis System, version 9.3 (SAS Institute Inc., Cary, North Carolina, United States) with the METADAS macro. for each study, we determined the sensitivity (true-positive rate); specificity (truenegative rate); LR+and LR- (estimated by calculating the ratio between the proportion of positive tests and that of negative tests in diseased vs. nondiseased subjects); and DOR (the LR+ divided by the $\mathrm{LR}^{-}$), with a $95 \%$ confidence interval. Those values were subsequently combined. We used the $\mathrm{I}^{2}$ statistic to assess the heterogeneity of the studies included. Meta-regression was used if there was high heterogeneity $\left(I^{2}>50 \%\right)$. We also constructed the SROC curve and calculated the respective AUC that serves a global measure of the test performance [14].

\section{Results}

\section{Articles selected}

In the initial search, 10,464 relevant articles were identified. After the titles, abstracts, and texts had been evaluated, 10,444 articles were excluded ( $\triangleright$ Fig. 2 ). Of the 20 remaining articles, six were excluded. Three articles were excluded because they did not provide the gold standard result required for construction of the $2 \times 2$ table [15-17]. Two articles were excluded because they evaluated buried BE after endoscopic treatment, one using OCT [18] and one using VLE [19], neither providing sufficient data for calculation of diagnostic accuracy. Another article was excluded because it evaluated feasibility of laser marking with VLE without allowing extraction of data for the calculation of diagnostic accuracy [20]. Therefore, the final sample comprised 14 articles.

\section{Study characteristics}

The 14 studies evaluated provided all of the necessary data to assess diagnostic accuracy of OCT and VLE, either by patient or by lesion, in identification of IM, dysplasia in general, HGD, and IMC in patients with BE. The studies evaluated a collective total of 721 patients (404 in the studies employing OCT and 317 in those employing VLE) and 1,565 areas of interest (984 in the studies employing OCT and 581 in those employing VLE), with $4 \%$ losses of the lesions being evaluated only with VLE ( $>$ Table 1). All of the studies selected were cross-sectional [21-34], OCT or VLE being performed sequentially after standard endoscopy: eight studies included patients undergoing BE surveillance [22, 24-26,29,30,33,34]; one included patients undergoing post-ablation surveillance [23]; two included patients undergoing surveillance after endoscopic eradication [21,31]; and three included patients undergoing surveillance after endoscopic mucosal resection [27, 28,32]. Eight of the studies employed an established diagnostic algorithm, defining true positives and true negatives [21,25-28,31,32,34], whereas three studies reported suspicious findings [22-24] and three did not establish a clear criterion [29,30,33].

Positive results were evaluated as follows: HGD and IMC were merged into a single diagnosis (neoplasia); LGD was considered an adjunct to HGD and IMC in the subgroup analysis of overall accuracy in the detection of dysplasia; and IM was evaluated in a separate subgroup analysis.

\section{Bias risk and applicability}

Among the eight VLE studies, risk of bias in patient selection was low in five studies (62.5\%) and high in three (37.5\%). Risk of bias in the index test (OCT- or VLE-guided targeted biopsy) was high in four studies (50.0\%) and low in two (25.0\%). Risk of bias in the gold-standard test (random biopsy) was low in all eight studies. Risk of bias in the QUADAS-2 flow and timing domain was low in six studies $(80.0 \%)$ and high in two $(25.0 \%)$. Applicability of the patient selection, index test, and goldstandard test was low in all eight studies ( $\mathbf{F i g . 3}$ ).

Among the six OCT studies, risk of bias in patient selection was low in five studies (83.3\%) and high in one (16.7\%). Risk of 


\begin{tabular}{l|c}
\hline OCT- Index Score & Pts \\
\hline Surface Maturation & \\
\hline Surface OCT signal weaker than sub- surface OCT signal & 0 \\
\hline Surface OCT signal equivalent to sub- surface OCT signal & 1 \\
\hline surface OCT signal stronger than subsurface OCT signal & 2 \\
\hline Gland Maturation & \\
\hline No irregularity, normal appearing; minimal number of smooth dilated glands & 0 \\
\hline Mild irregularity, glands were smaller or large and irregularly shaped; dilated glands & 1 \\
\hline Moderate/severe irregularity, dilated glands were highly asymmetric or contained debris within the gland lumen & 2 \\
\hline
\end{tabular}

\section{OCT Criteria for SIM}

1) Absence of the layered structure of normal squamous epithelium and the vertical "pit and crypt" morphology of normal gastric mucosa

2) Disorganized architecture with irregular mucosal surface

3) Presence of submucosal glands of low reflectance below the epithelial surface or invaginations through the epithelium

b

Computer-Aided Detection

Clinically inspired features

1) Layering

2) Layering and signal decay statistics

3) Signal intensity distribution

c

VLE Prediction Score

Layering

Layering present $(>50 \%)$

Lack of layering $(<50 \%)$

VLE Surface Signal

Surface signal < subsurface

Surface signal $=$ subsurface $(>50 \%)$

Surface signal $>$ subsurface $(<50 \%)$

\section{Gland Architecture}

Irregular glands ( $0-5$ glands)

Irregular glands (>5 glands)

d

\section{VLE-Diagnostic Algorithm}

Mucosal Layer (ML):

1) Partial Effacement (ML $>2 \mathrm{~mm}$ in $50 \%$ of the scan)

$>5$ atypical glands $=$

$\leq 5$ atypical glands $=$

\section{2) Complete Effacement (ML over $<50 \%$ of the scan)}

Surface $\leq$ Subsurface intensity $=$

Surface $>$ Subsurface intensity $=$

Fig. 1 Optical coherence tomography (OCT) and volumetric laser endomicroscopy (VLE) scores. 


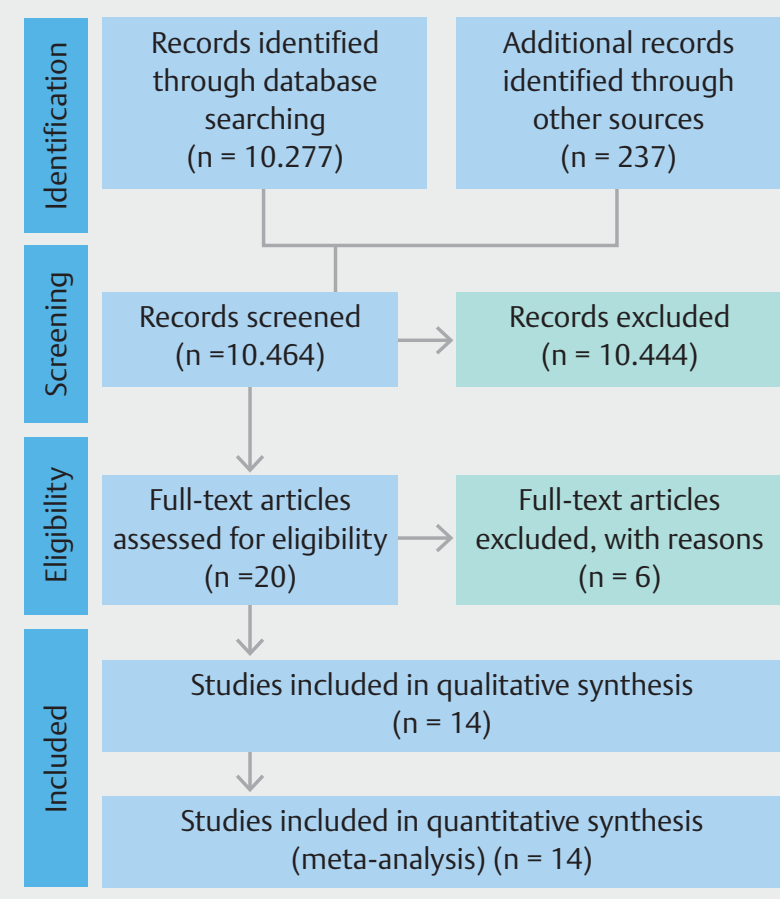

- Fig. 2 Study selection process.

bias in the index test was low in five studies (83.3\%) and unclear in one $(16.7 \%)$. Risk of bias in the gold-standard test was low in all six studies. Risk of bias in the QUADAS-2 flow and timing domain was low in five studies (83.3\%) and high in one $(16.7 \%)$. Applicability of the patient selection, index test, and goldstandard test was low all six studies ( $\vee$ Fig.4).

\section{Results of individual studies and data synthesis}

Analyses of the VLE findings, including subgroup analyses of diagnostic accuracy for detection of dysplasia in general (LGD, HGD, or IMC) and for detection of HGD/IMC, were performed by patient and by lesion. Analyses of the OCT findings, including subgroup analyses of diagnostic accuracy for detection of IM, dysplasia in general, and HGD/IMC, were performed only by lesion.

\section{OCT findings}

\section{HGD/IMC}

Per-lesion analysis of diagnostic accuracy for detection of HGD/ IMC was based on four articles [21, 24,33,34]. As depicted in - Fig. 5, that analysis yielded a pooled sensitivity of $89 \%$, pooled specificity of $91 \%$, pooled LR+of 9.6 (95\% Cl: $1.1-$ 86.4), pooled LR- of 0.12 (95\% Cl: $0.02-0.57$ ), DOR of 81 (95\% Cl: 4-1702), and SROC AUC of 0.95 (95\% Cl: $0.82-$ 0.99). In addition, the overall $\mathrm{I}^{2}$ value was 83 (95\% Cl: $64-$ $100)$, indicating high heterogeneity. Therefore, we adjusted the meta-regression models to identify possible sources of heterogeneity among the estimates. To that end, the following were considered as predictor variables: use of diagnostic algo- rithms; use of conventional OCT; real-time evaluation; and offline evaluation. As can be seen in $>$ Fig. 6, specificity was significantly higher in articles that employed real-time evaluation than in those that employed offline evaluation $(P=0.020)$.

The per-lesion analysis for real-time (in vivo) evaluation was based on three articles [24,33,34]. That analysis yielded a pooled sensitivity of $79 \%(95 \% \mathrm{Cl}: 56-92 \%)$, pooled specificity of $94 \%$ (95\% Cl: $36-99 \%$ ), pooled LR+of 15.6 (95\% Cl: $0.49-$ 490), pooled LR- of 0.21 ( $95 \% \mathrm{Cl}: 0.08-0.57)$, and DOR of 73.20 (95\% Cl: $1.09-489)$, denoting a drop in sensitivity due to the exclusion of one article [21], which, in isolation, had a sensitivity of $100 \%$ due to better image quality.

\section{Detection of dysplasia in general}

Analysis of diagnostic accuracy for detection of dysplasia in general was based on three articles [21,24,33]. Due to the small number of cases evaluated in those three articles, it was feasible to calculate only the main measures of accuracy or diagnostic performance (i.e., it was not possible to calculate an AUC). Therefore, the analysis yielded a pooled sensitivity of $89 \%(95 \% \mathrm{Cl}: 69-96 \%)$, pooled specificity of $95 \%(95 \% \mathrm{Cl}$ : $48-99 \%$ ), pooled LR+of 19.85 (IC $0.93-422$ ), pooled LR- of $0.11(95 \% \mathrm{Cl}: 0.033-0.38)$ and DOR of $175.74(95 \% \mathrm{Cl}$ : $3.425-9015.73)$.

\section{IM}

Diagnosis of IM was analyzed based on two articles [25, 26]. Again, due to the small number of cases, only the main measures of accuracy were evaluated. For identification of IM, OCT had a pooled sensitivity of $92 \%$ (95\% Cl: $66-98 \%)$, a pooled specificity of $81 \%$ ( $95 \% \mathrm{Cl}: 56-93 \%)$, pooled LR+of $5.06(95 \%$ Cl: $3.09-15.60)$, pooled LR- of 0.091 (IC 95\% Cl: $0.01-0.59$ ), and DOR of 55.58 (95\% Cl: $3.09-999.49)$.

\section{VLE findings}

\section{Findings by lesion}

Per-lesion analysis of diagnostic accuracy for detection of HGD/ IMC was based on five articles [28-32]. That analysis yielded a pooled sensitivity of $85 \%$ ( $95 \% \mathrm{Cl}: 75-91 \%)$, pooled specificity of $73 \%$ ( $95 \% \mathrm{Cl}: 52-87 \%)$, pooled LR+ of 3.2 (95\% Cl: $1.6-6.4)$, pooled LR- of 0.21 (95\% Cl: $0.11-0.39)$ and DOR of $15(95 \% \mathrm{Cl}$ : $4-53)$. As shown in $>$ Fig. 7, the SROC AUC was $0.87(95 \% \mathrm{Cl}$ : $0.66-0.96)$ and the $\mathrm{I}^{2}$ was 53 (95\% Cl: $\left.0-100\right)$, indicating moderate heterogeneity. Meta-regression was performed to identify possible sources of heterogeneity. Sample size, positivity criterion established, age, real-time evaluation, and proportion of males were considered as predictor variables. As can be seen in - Fig. 8, specificity was significantly lower in articles that employed real-time evaluation than in those that employed offline evaluation $(P=0.010)$.

\section{Findings by patient}

Per-patient analysis of diagnostic accuracy for real-time (in vivo) detection of HGD/EAC was based on three articles [22, $23,27]$. Once again, due to the small number of cases, only the main measures of accuracy were evaluated. That analysis had a 


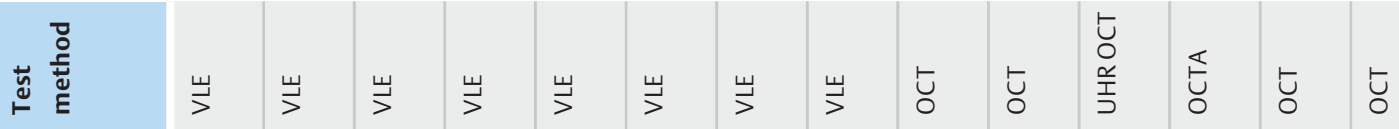

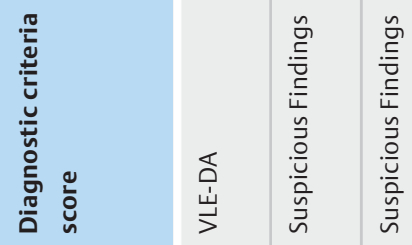

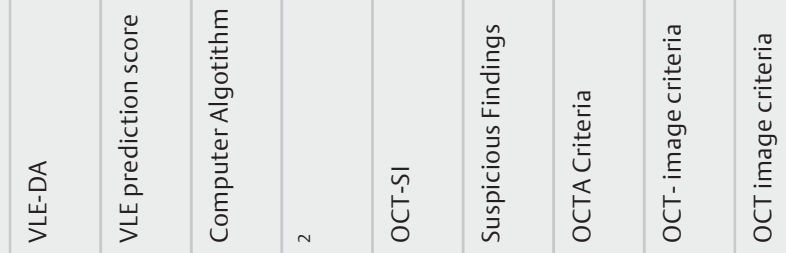

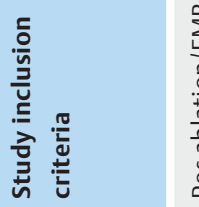

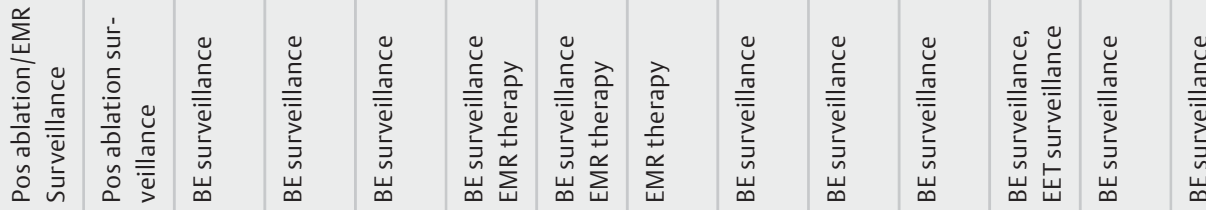

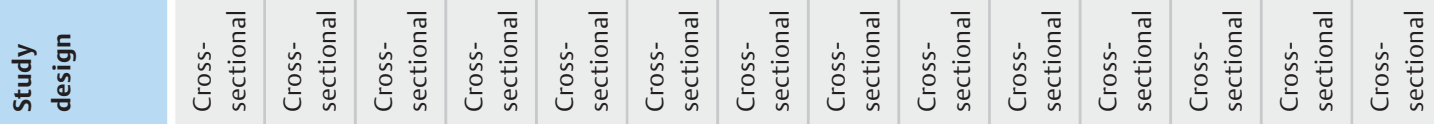

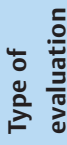

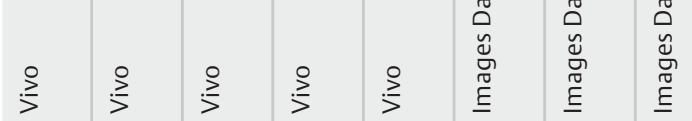

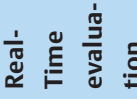

(2)

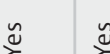

$\check{2}$

2

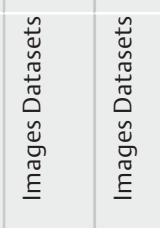

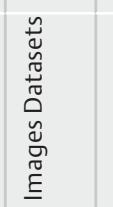

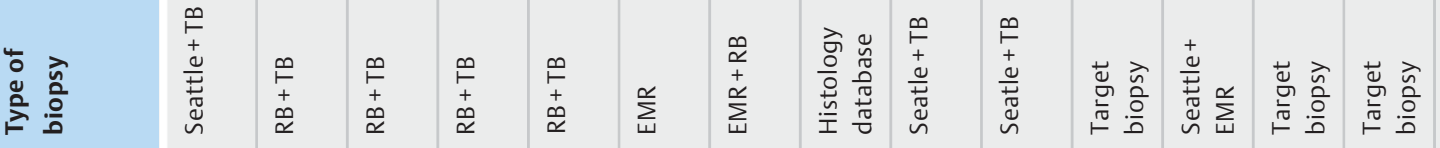

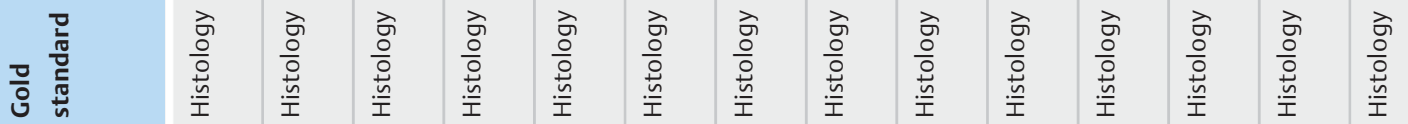

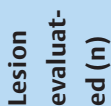

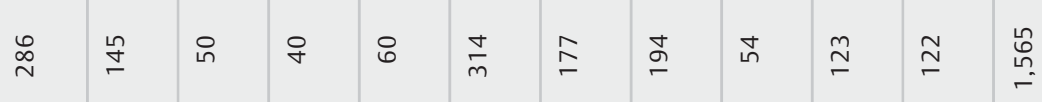

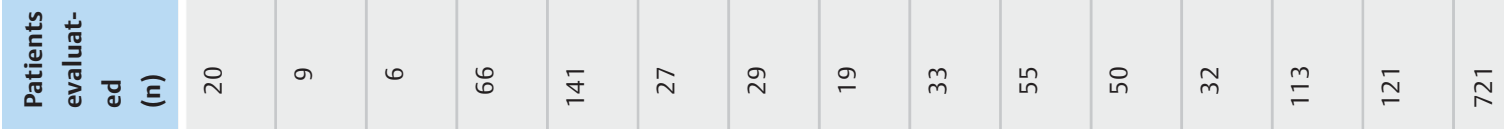

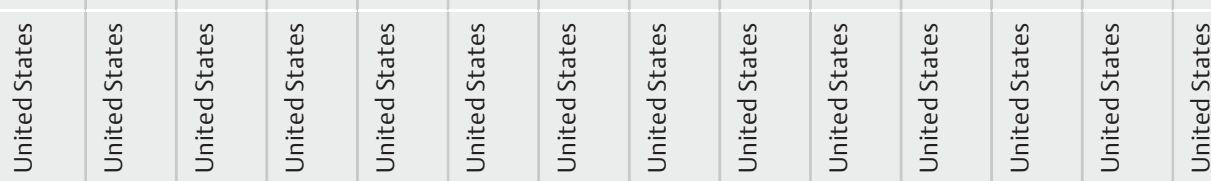

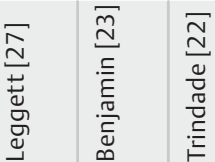

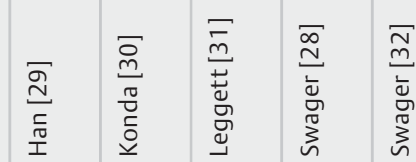

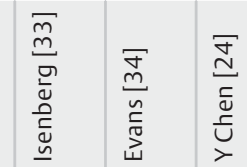

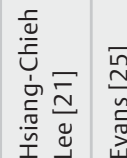

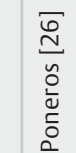

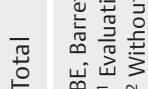



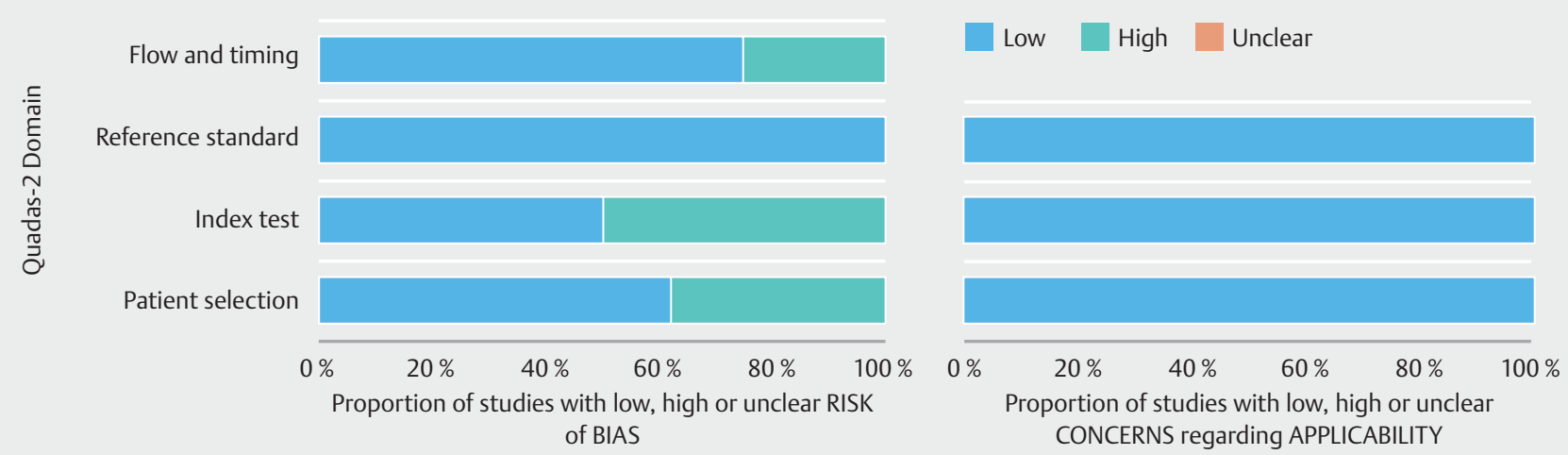

Dig.3 Quality assessment of diagnostic accuracy studies, (QUADAS-2) assessment of risk of bias in VLE.

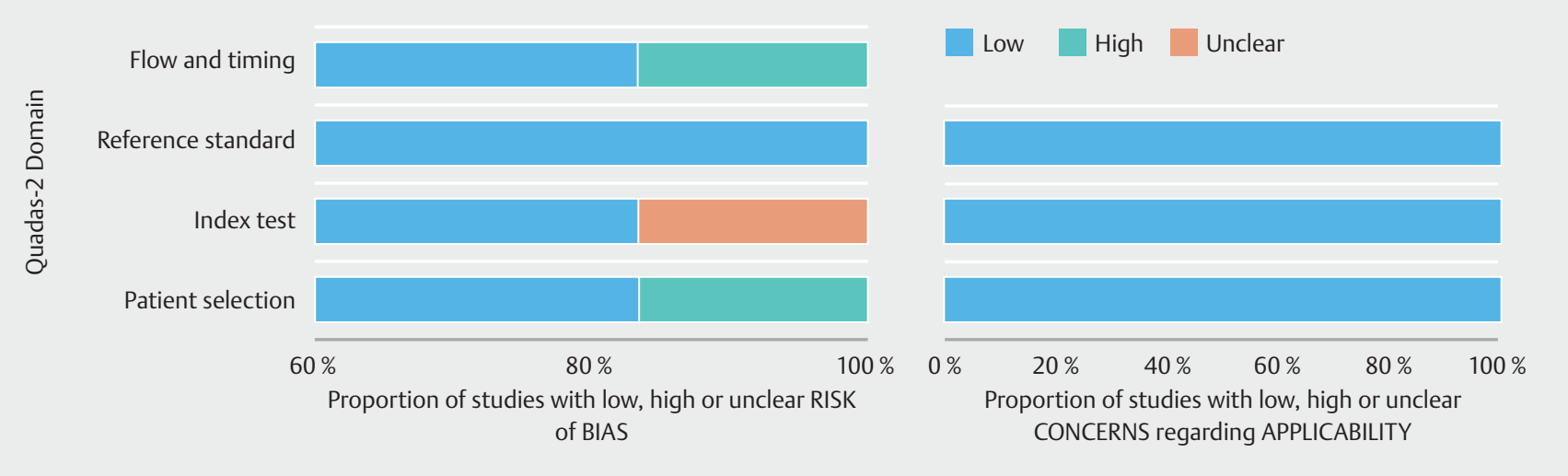

Fig.4 Quality assessment of diagnostic accuracy studies, (QUADAS-2) assessment of risk of bias in OCT.

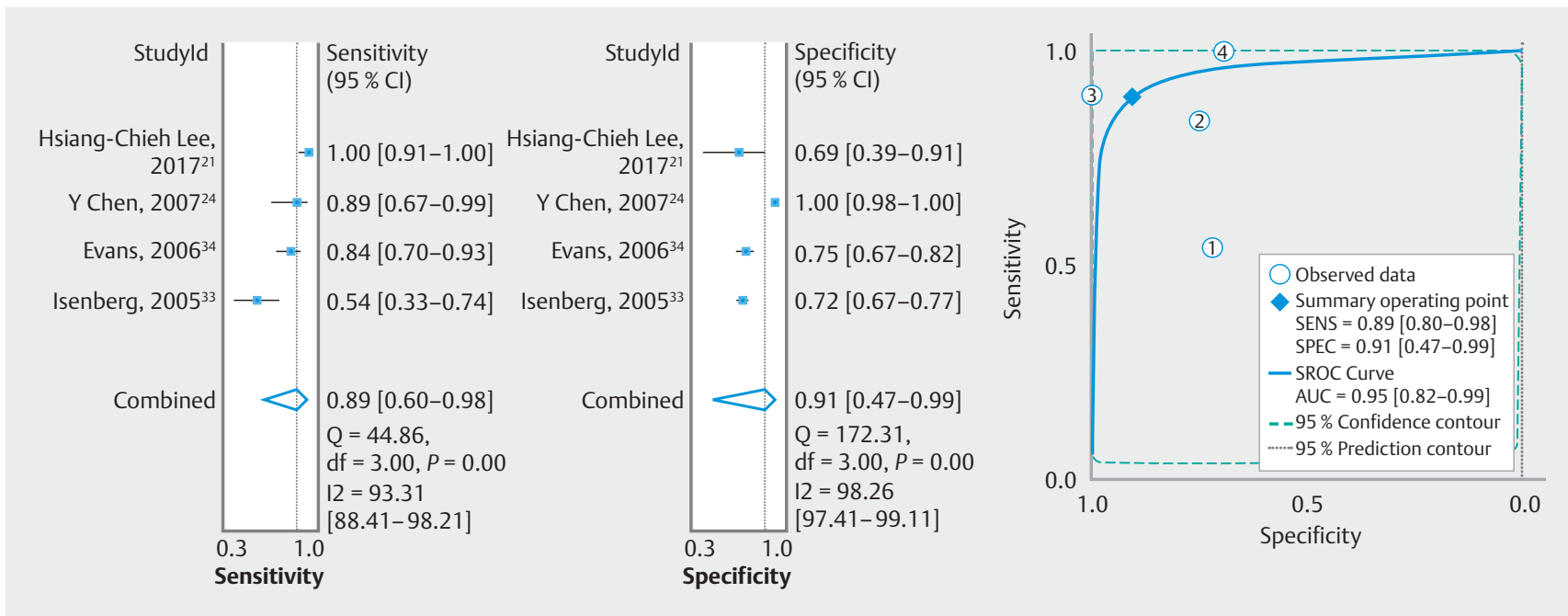

- Fig. 5 Forest plot of optical coherence tomography (OCT) sensitivity and specificity for detection of high-grade dysplasia/intramucosal carcinoma, by lesion and summary receiver operating characteristic (SROC) curve and area under the curve (AUC).

pooled sensitivity of $100 \%$, pooled specificity of $55 \%(95 \% \mathrm{Cl}$ : $29-79 \%)$, pooled LR+of 2.27 (95\% Cl: $1.22-4.19)$, pooled LRof 0.00 , and DOR of 1.028 .
Findings by patient and by lesion

Per-lesion and per-patient analyses of diagnostic accuracy for detection of HGD/IMC were based on eight articles [22, 23, 27 -32]. As shown in $>$ Fig. 9, those analyses had a pooled sen- 


\section{Univariable meta-regression and subgroup analysis}

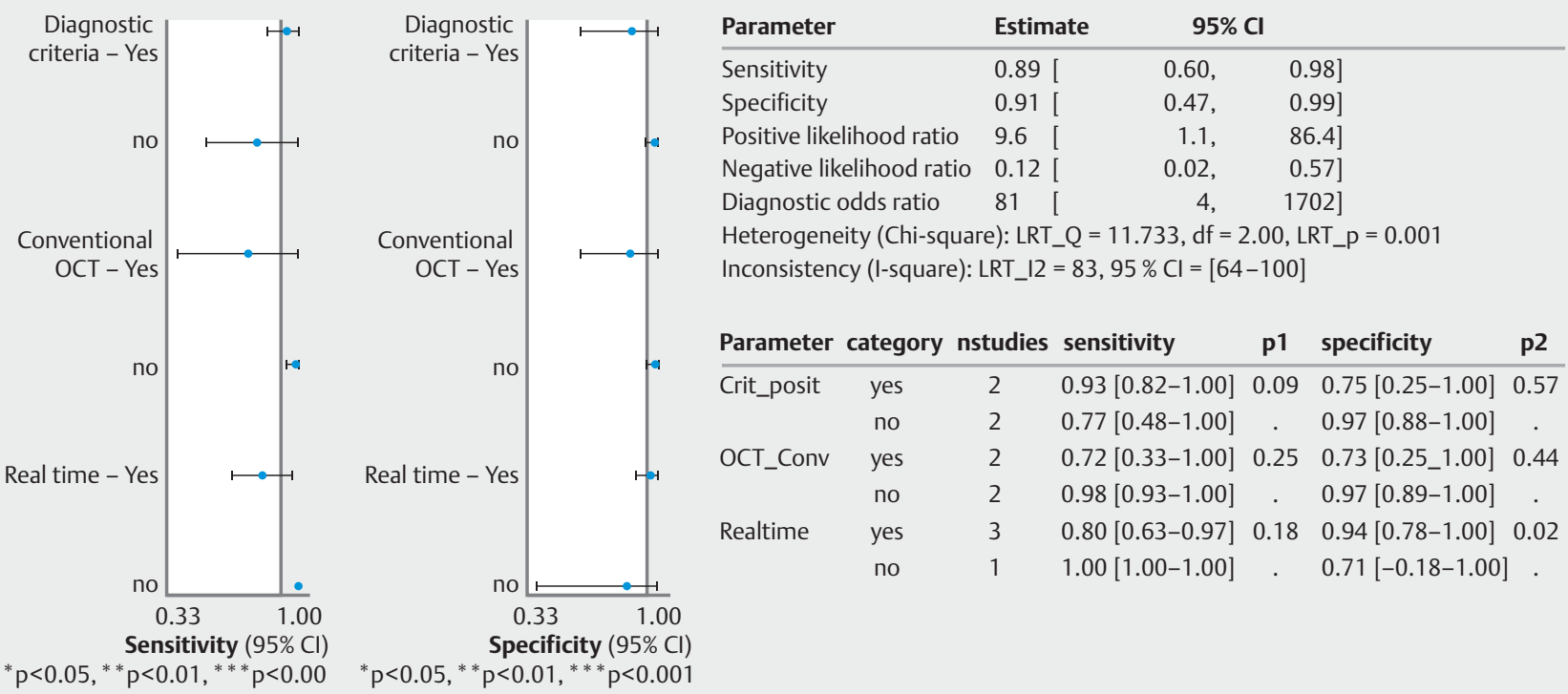

Fig. 6 Sensitivity and specificity of optical coherence tomography (OCT), estimated by meta-regression.

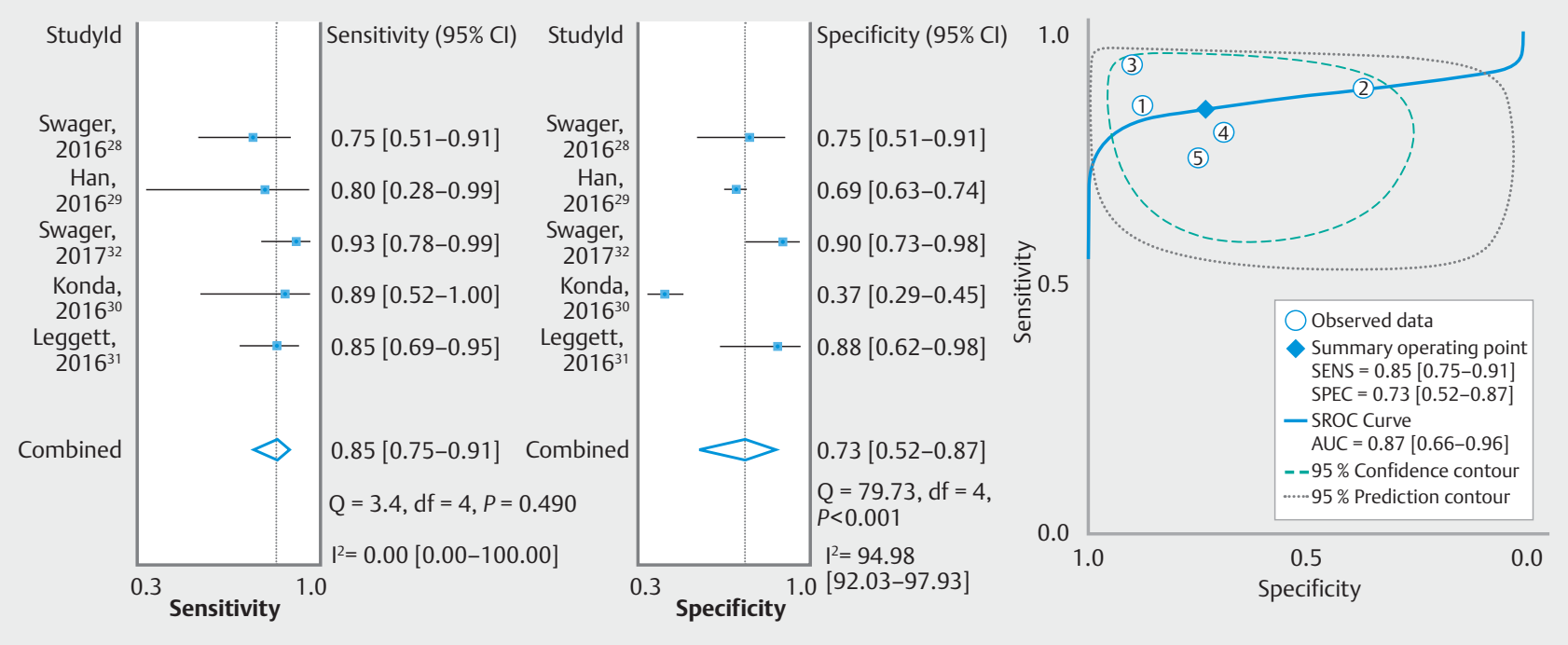

Fig. 7 Volumetric laser endomicroscopy (VLE) sensitivity and specificity for detection of high-grade dysplasia/intramucosal carcinoma, by lesion and summary receiver operating characteristic (SROC) curve and area under the curve (AUC).

sitivity of $87 \%(95 \% \mathrm{Cl}: 77-93 \%)$, pooled specificity of $68 \%$ (95\% Cl: $51 \%-82 \%)$, pooled LR+of 2.7 (95\% Cl: $1.6-4.5)$, pooled LR- of 0.20 (95\% Cl: $0.10-0.37)$, DOR of $14(95 \% \mathrm{Cl}$ : 5-38), and an SROC AUC of 0.87 (95\% Cl: 0.67-0.96). The $\mathrm{I}^{2}$ was 54 (95\% Cl: $0-100)$, indicating moderate heterogeneity, and we adjusted the meta-regression models accordingly. Sample size, positivity criterion established, age, real-time evaluation, and proportion of males were considered as predictor variables. As can be seen in > Fig. 10, specificity was again significantly lower in articles that employed real-time evaluation than in those that employed offline evaluation $(P=0.010)$.

\section{Detection of dysplasia in general}

Analysis of diagnostic accuracy for detection of dysplasia in general was based on four articles [23, 27, 29, 30]. That analysis yielded a pooled sensitivity of $93 \%$ (95\% Cl: $80 \%-98 \%)$, pooled specificity of $54 \%(95 \% \mathrm{Cl}: 37-70 \%)$, pooled LR+of $2.0(95 \% \mathrm{Cl}$ : $1.4-2.8)$, pooled LR- of $0.12(95 \% \mathrm{Cl}: 0.04-0.35)$, and DOR of 16 (95\% Cl: 6-46). As shown in > Fig. 11, the SROC AUC was 0.85 (95\% Cl: $0.81-0.88)$ and the overall $\mathrm{I}^{2}$ was $42(95 \% \mathrm{Cl}$ : $0-100)$, indicating mild heterogeneity. 


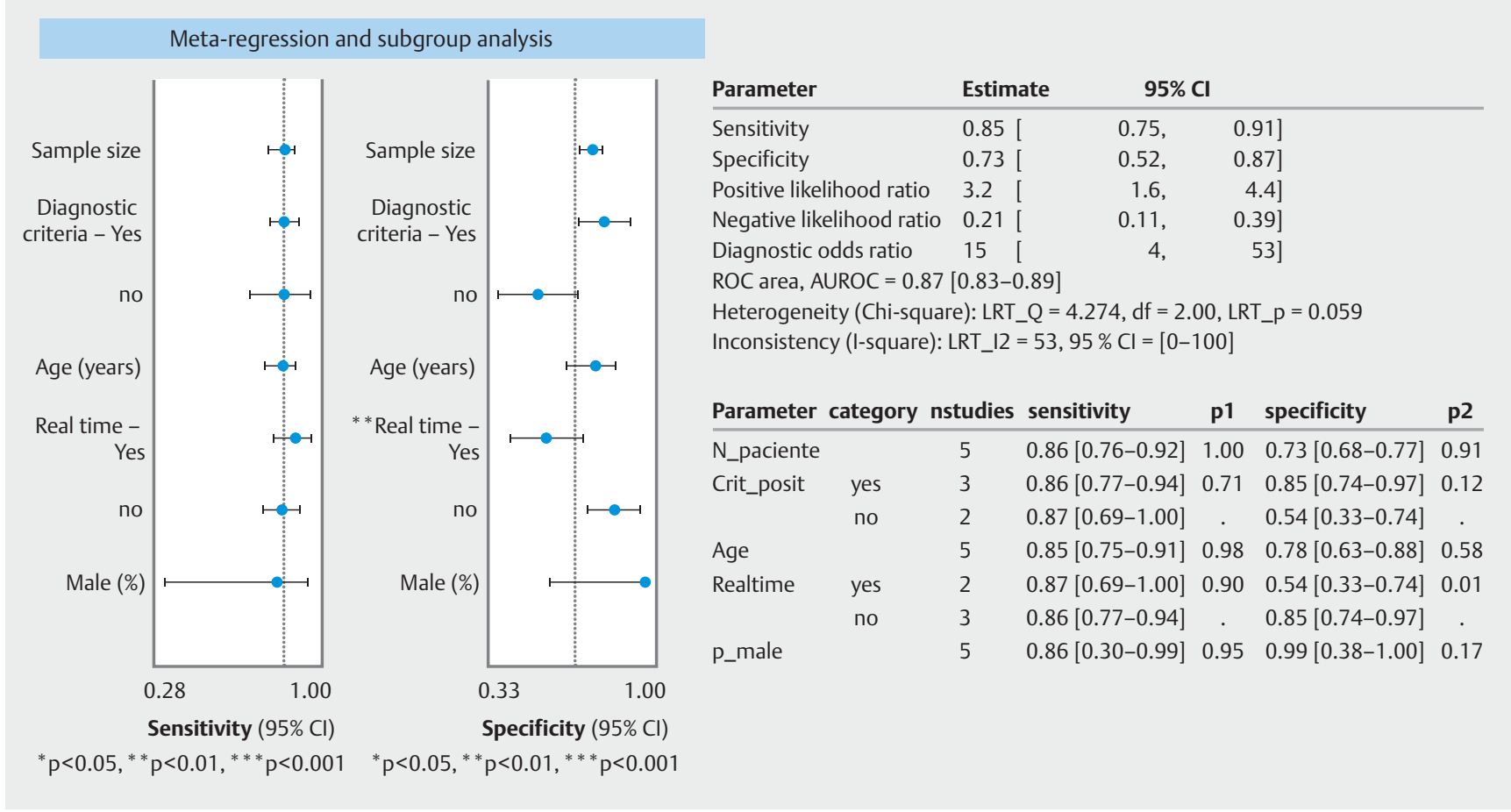

Fig. 8 Sensitivity and specificity of volumetric laser endomicroscopy (VLE), by lesion, estimated by meta-regression.

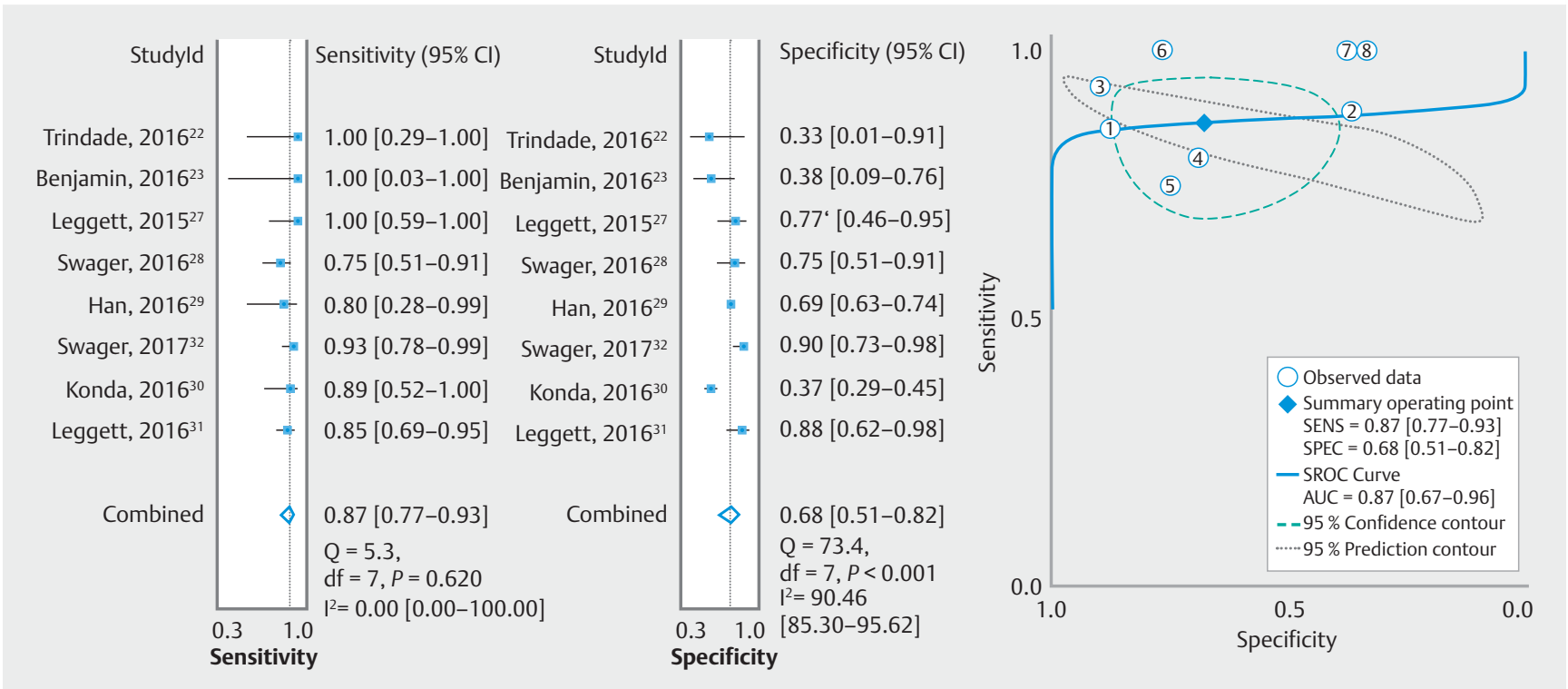

- Fig. 9 Forest plot of volumetric laser endomicroscopy (VLE) sensitivity and specificity for detection of high-grade dysplasia/intramucosal carcinoma, by lesion and by patient and summary receiver operating characteristic (SROC) curve and area under the curve (AUC).

\section{Discussion}

Because of the importance of identifying esophageal cancer in its early stages, when a cure is still possible, various advanced diagnostic imaging methods are being studied. Lack of a recommendation for routine use of such methods in surveillance of patients with $\mathrm{BE}$ is due in part to the fact that their use in daily practice has yet to be validated in large studies or does not meet the threshold established for surveillance of patients with BE in the American Society for Gastrointestinal Endoscopy Preservation and Incorporation of Valuable Endoscopic Innovations (PIVI) initiative [3]. Neither OCT nor VLE has been validated.

Use of OCT and VLE not only facilitates identification and differentiation of lesions by distinguishing between benign and 


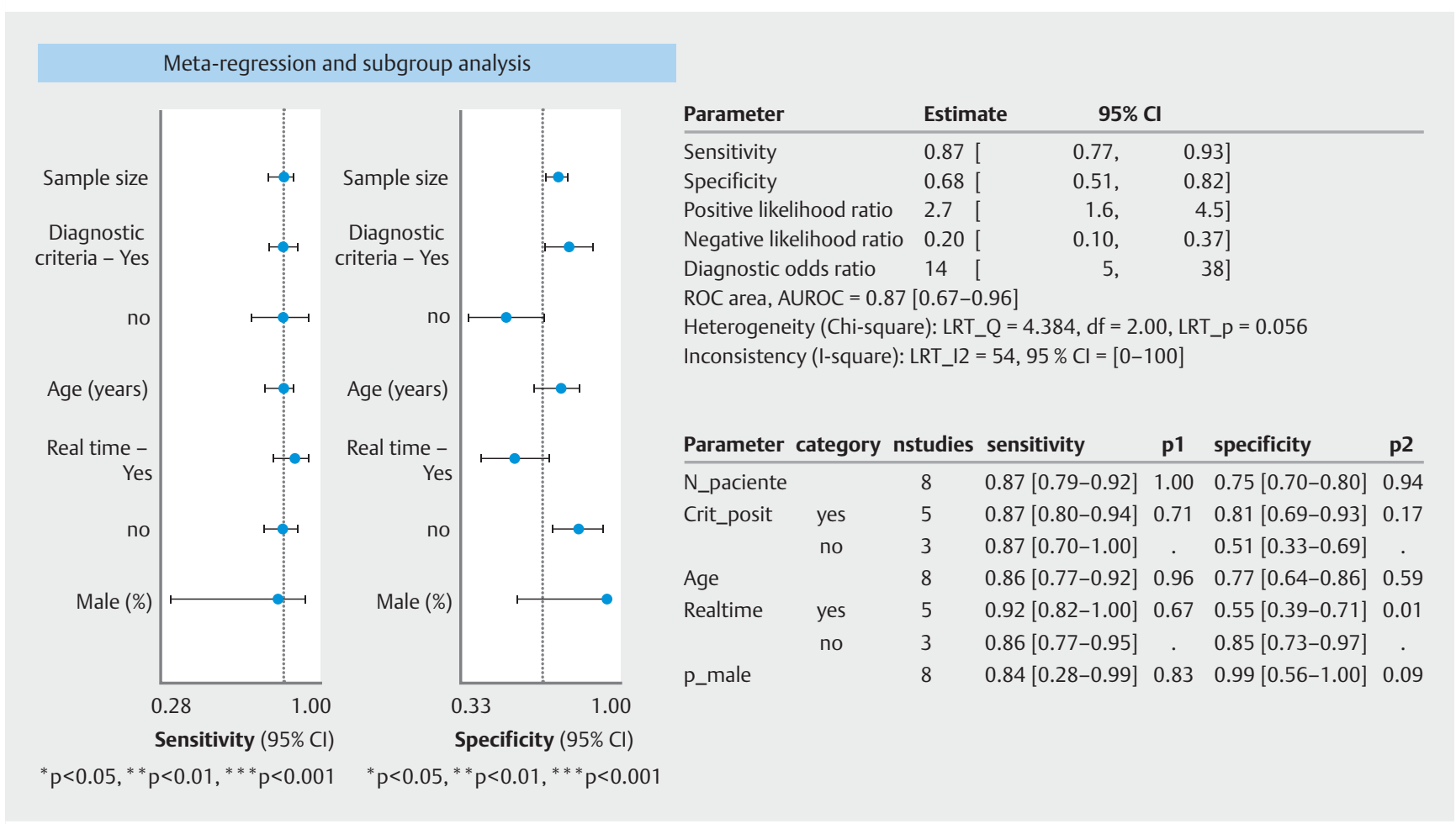

Fig. 10 Sensitivity and specificity of volumetric laser endomicroscopy (VLE), by lesion and by patient, estimated by meta-regression.

malignant characteristics by direct microscopic investigation but also allows evaluations to be performed simultaneously with the usual endoscopic examination, playing the role of an "optical biopsy," identifying suspicious areas that can be biopsied or resected under guidance, thus reducing sampling errors and improving overall diagnostic sensitivity [35].

Perhaps the greatest challenge in BE surveillance identifying dysplasia and neoplasia in long BE segments. If such abnormalities are diagnosed during surveillance, ablative endoscopic therapy can be performed. In a recent study of patients with BE, Alshelleh et al. [36] demonstrated a significant statistically significant difference between VLE and VLEL, as compared by using the Seattle protocol, in detection of HGD (14\% vs. $1 \%$; $P$ $=0.001)$ and IMC ( $11 \%$ vs. $1 \% ; P=0.003)$, supporting use of VLE at teaching facilities. In a study conducted by Leggett et al. [31], the use of VLE and the VLE diagnostic algorithm showed a sensitivity of $86 \%$ and a specificity of $88 \%$ for detection of HGD/IMC. In our meta-analysis, we showed that VLE by lesion had a sensitivity of $85 \%$ and a specificity of $73 \%$ for the detection of HGD/IMC, with an AUC SROC of 0.87 , with values similar to those published previously, corroborating findings of the studies cited above $[31,36]$ and showing that VLE can differentiate neoplastic lesions from non-neoplastic lesions in patients with $\mathrm{BE}$. However, in the per-patient analysis of detection of HGD/IMC, we found that VLE showed a higher sensitivity (100\%) and a lower specificity (55\%). That is because, although most of the VLE studies analyzed reported high sensitivity, two presented low specificity $[22,23]$. With the exception of the Leggett et al. study [27], in which the authors showed a specificity of $76.9 \%$, none of the articles evaluated employed a stand- ardized diagnostic algorithm. That demonstrates the importance of standardizing a diagnostic algorithm for true-positive results. That also serves to explain results obtained in the analysis of detection of dysplasia in general, in which VLE was found to have a sensitivity of $93 \%$ and a specificity of $54 \%$. That demonstrates that creation of scores improves detection of neoplastic lesions. However, LGD is considered a difficult diagnosis to make on the basis of imaging findings as well as on the basis of pathology findings [28], because presence of inflammation can confound diagnosis of dysplasia [33].

In a previous systematic review, Kholi et al. [12] reported that, for identification of IM, OCT has a sensitivity of $81 \%$ to $97 \%$ and a specificity of $57 \%$ to $92 \%$, whereas it has a sensitivity of $68 \%$ to $83 \%$ and a specificity of $75 \%$ to $82 \%$ for identification of dysplasia and early-stage cancer. In addition to studies employing first-generation OCT, we included studies employing the latest generation OCT, which makes it possible to evaluate microvasculature and differentiate more easily between LGD and neoplasia, as well as to extract data for subgroup analyses.

As recommended in the most recent guideline [2], endoscopic therapy should be considered the gold-standard treatment modality for patients with LGD. Complete elimination of $\mathrm{IM}$ is also recommended after successful endoscopic therapy in patients with HGD or IMC. Therefore, we performed a subgroup analysis to calculate accuracy of OCT for identification of IM, although such an analysis was not possible for VLE. When identified in the VLE or OCT studies, LGD was considered a positive result and was grouped with $\mathrm{HGD} / \mathrm{IMC}$ for the analysis of the detection of dysplasia in general. 


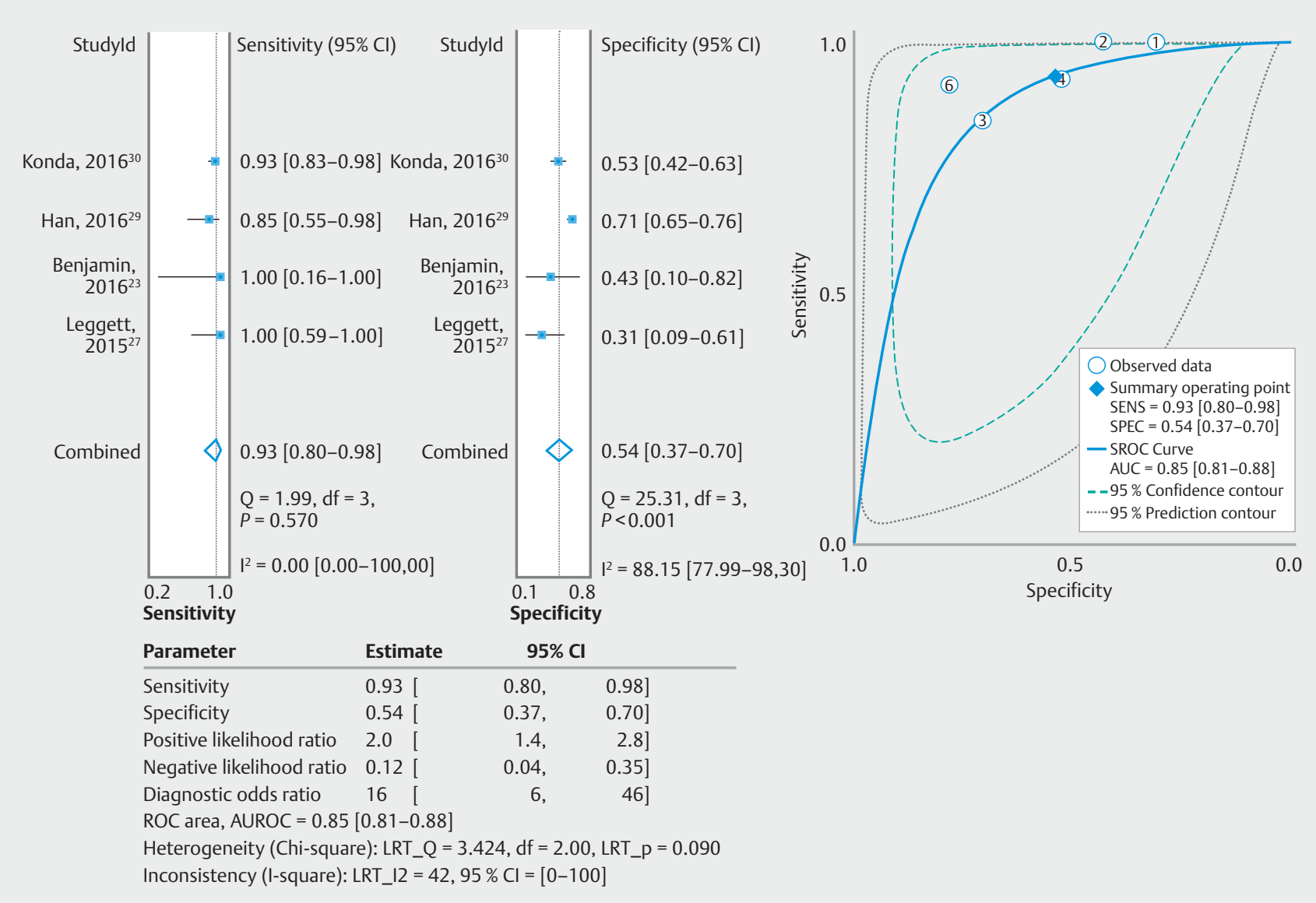

- Fig. 11 Forest plot of volumetric laser endomicroscopy (VLE) sensitivity and specificity for dysplasia and summary receiver operating characteristic (SROC) curve and area under the curve (AUC).

For identification of HGD/IMC, OCT had a pooled sensitivity of $89 \%$ and specificity of $91 \%$, values close to those reported in the review article authored by Kholi et al. [12], as well as an SROC AUC of 0.95 . These results indicate that OCT has a strong ability to differentiate neoplasms from non-neoplasms in patients with BE. For identification of IM, OCT had a sensitivity and specificity of $92 \%$ and $81 \%$, respectively, again corroborating the values reported previously.

Our study has certain limitations, some related to technology and some related to methodology. The OCT studies were not standardized in terms of the technology employed, given that it is constantly evolving. The most recent devices have higher resolution and therefore tend to have a higher rate of lesion detection. Two other factors that affected diagnostic performance were non-standardization or absence of a diagnostic algorithm for positivity in the studies and the manner in which the evaluations were made (in real time or offline). However, although these factors were present in the VLE and OCT studies. In the OCT only one that was significant in relation to specificity was the real-time evaluation. In fact, lower accuracy in evaluation of image datasets (offline evaluation) is likely due to the appearance of the tissues, which is different in evaluation of images in real time [28]. The type of comparison made in our review allows us to confirm that the best accuracy is obtained with real-time evaluation.

Among the OCT studies, there was only one that did not establish a diagnostic algorithm as a criterion, that one study having little effect on the heterogeneity. That was not so for the four VLE studies that did not establish a diagnostic algorithm or only reported suspicious findings. All of those studies involved real-time (in vivo) interpretation, which was found to be a significant, indirect predictor of heterogeneity, with a lower specificity for detection of HGD/IMC, as previously stated. Algorithms for automated analysis of VLE data can make a valuable contribution to their interpretation [32], given the large amount of data to be analyzed in real time. Van der Sommen et al. [37] investigated the potential of algorithm-based computer-aided detection (CADe) for identification of neoplasms. The authors found that the ex vivo use of VLE and CADe had an AUC of $0.90-0.93$, versus 0.81 for specialist physicians, showing that computer-aided methods can achieve considerably better performance than do human observers.

Another feature that improves VLE performance is use of VLEL, because it allows the lesions to be delimited and direct histological samples to be obtained with adequate safety margins, thus improving detection and delineation of neoplastic lesions in BE [20]. Alshelleh et al. [36] demonstrated that, in 
groups of patients undergoing VLEL surveillance in accordance with the Seattle protocol, VLEL showed statistically higher rates of detection of dysplasia in general (19.6\% vs. $33.7 \%$; OR $=2.1$; $P=0.03)$.

Following the diagnostic thresholds recommended in the PIVI initiative [35] for detection of HGD/IMC in patients undergoing BE surveillance, it would be necessary to achieve a sensitivity $\geq 90 \%$, a negative predictive value $\geq 98 \%$, and a specificity $\geq 80 \%$ to replace use of random biopsies with that of targeted biopsies. In the current review, OCT had thresholds close to or above those targets, with a sensitivity of $89 \%$ and specificity of $91 \%$. However, the analyses were made by lesions, due to the lack of per-patient studies. When the analysis was limited to studies employing real-time evaluation, sensitivity and specificity were $79 \%$ and $94 \%$, respectively. Therefore, OCT does not meet the thresholds needed to replace the current surveillance protocol.

In the per-patient analysis of identification of HGD/IMC, VLE had a pooled sensitivity of $100 \%$ and a negative predictive value of $100 \%$, although it had a specificity of only $55 \%$. Therefore, it is still not yet possible to replace random biopsies with targeted biopsies, given the current state of the technology.

It is too early to assess the in vivo diagnostic performance of VLE, given that there are limited data available. Multicentric studies, with adequate standardization of screening criteria and diagnostic algorithms, as well as incorporation of VLEL in the studies are needed to lay the groundwork for its broader use in clinical contexts.

\section{Conclusions}

OCT and VLE are both effective methods for differentiating and detecting IM, dysplasia, and neoplasia in patients with BE. Concomitant use of these technologies and the current surveillance protocol could improve the rate of detection of dysplasia and neoplasia.

\section{Competing interests}

None

\section{References}

[1] Pohl H, Welch HG. The role of overdiagnosis and reclassification in the marked increase of esophageal adenocarcinoma incidence. J Natl Cancer Inst 2005; 97: 142-146

[2] Shaheen NJ, Falk GW, Iyer PG et al. ACG Clinical Guideline: diagnosis and management of Barrett's esophagus. Am J Gastroenterol 2016; 111: $30-50$; quiz 51

[3] Thosani N, Abu Dayyeh BK et al. ASGE Technology Committee systematic review and meta-analysis assessing the ASGE Preservation and Incorporation of valuable endoscopic innovations thresholds for adopting real-time imaging-assisted endoscopic targeted biopsy during endoscopic surveillance. Gastrointest Endosc 2016; 83: 684 698.e7
[4] Sharma P, Brill J, Canto M et al. White paper AGA: advanced imaging in Barrett's esophagus. Clin Gastroenterol Hepatol 2015; 13: 2209 2218

[5] Tearney G], Brezinski ME, Bouma BE et al. In vivo endoscopic optical biopsy with optical coherence tomography. Science 1997; 276: 2037-2039

[6] Sivak MV, Kobayashi K, Izatt JA et al. High-resolution endoscopic imaging of the $\mathrm{Gl}$ tract using optical coherence tomography. Gastrointest Endosc 2000; 51: 474-479

[7] Tsai T-H, Lee H-C, Ahsen OO et al. Ultrahigh speed endoscopic optical coherence tomography for gastroenterology. Biomed Opt Express 2014; 5: 4387-4404

[8] Aguirre AD, Chen Y, Bryan B et al. Cellular resolution ex vivo imaging of gastrointestinal tissues with optical coherence microscopy. J Biomed Opt 2010; 15: 016025

[9] Tsai T-H, Zhou C, Tao YK et al. Structural markers observed with endoscopic 3-dimensional optical coherence tomography correlating with Barrett's esophagus radiofrequency ablation treatment response (with videos). Gastrointest Endosc 2012; 76: 1104-1112

[10] Wolfsen HC. Volumetric laser endomicroscopy in patients with Barrett esophagus. Gastroenterol Hepatol (NY) 2016; 12: 719-722

[11] Suter MJ, Gora MJ, Lauwers GY et al. Esophageal-guided biopsy with volumetric laser endomicroscopy and laser cautery marking: a pilot clinical study. Gastrointest Endosc 2014; 79: 886- 896

[12] Kohli DR, Schubert ML, Zfass AM et al. Performance characteristics of optical coherence tomography in assessment of Barrett's esophagus and esophageal cancer: systematic review. Dis esophagus Off J Int Soc Dis Esophagus 2017; 30: $1-8$

[13] Whiting PF, Rutjes AWS, Westwood ME et al. QUADAS-2: a revised tool for the quality assessment of diagnostic accuracy studies. Ann Intern Med 2011; 155: 529-536

[14] Higgins JPT, Thompson SG, Deeks JJ et al. Measuring inconsistency in meta-analyses. BMJ 2003; 327: 557-560

[15] Kamboj AK, Kahn A, Wolfsen HC et al. Volumetric laser endomicroscopy interpretation and feature analysis in dysplastic Barrett's esophagus. J Gastroenterol Hepatol 2018; 33: 1761 - 1765

[16] Smith MS, Cash BD, Trindade AJ. Dysplasia suggested by suspicious findings on volumetric laser endomicroscopy may be detected on initial or follow-up endoscopy. Gastrointest Endosc 2017; 85: AB78

[17] Cash BD, Joshi V, Wolfsen HC et al. Absence of suspicious findings on volumetric laser endomicroscopy strongly predicts histopathologic complete remission of dysplasia and intestinal metaplasia in patients after visual eradication of Barrett's esophagus. Gastrointest Endosc 2016; 83: AB123

[18] Hatta W, Uno K, Koike T et al. Feasibility of optical coherence tomography for the evaluation of Barrett's mucosa buried underneath esophageal squamous epithelium. Dig Endosc 2016; 28: 427-433

[19] Swager AF, Boerwinkel DF, De Bruin DM et al. Detection of buried Barrett's glands after radiofrequency ablation with volumetric laser endomicroscopy. Gastrointest Endosc 2016; 83: 80-86

[20] Swager A-F, de Groof A], Meijer SL et al. Feasibility of laser marking in Barrett's esophagus with volumetric laser endomicroscopy: first-inman pilot study. Gastrointest Endosc 2017; 86: 464-472

[21] Lee HC, Ahsen OO, Liang K et al. Endoscopic optical coherence tomography angiography microvascular features associated with dysplasia in Barrett's esophagus (with video). Gastrointest Endosc 2017; 86: $476-484 . e 3$

[22] Trindade A], George B], Berkowitz J et al. Volumetric laser endomicroscopy can target neoplasia not detected by conventional endoscopic measures in long segment Barrett's esophagus. Endosc Int open 2016; 4: E318-22 
[23] Benjamin T, Shakya S, Thota PN. Feasibility of volumetric laser endomicroscopy in Barrett's esophagus with dysplasia and in post-ablation surveillance. J Gastrointestin Liver Dis 2016; 25: 407- 408

[24] Chen Y, Aguirre AD, Hsiung PL et al. Ultrahigh resolution optical coherence tomography of Barrett's esophagus: preliminary descriptive clinical study correlating images with histology. Endoscopy 2007; 399: 599-605

[25] Evans JA, Bouma BE, Bressner J et al. Identifying intestinal metaplasia at the squamocolumnar junction by using optical coherence tomography. Gastrointest Endosc 2007; 65: 50 - 56

[26] Poneros JM, Brand S, Bouma BE et al. Diagnosis of specialized intestinal metaplasia by optical coherence tomography. Gastroenterology 2001; 120: 7-12

[27] Leggett CL, Chan DK, Gorospe EC et al. Tu1542 diagnostic performance of in-vivo volumetric laser endomicroscopy for detection of Barrett's esophagus dysplasia. Gastrointest Endosc 2015; 81: AB502

[28] Swager AF, Tearney G], Leggett CL et al. Identification of volumetric laser endomicroscopy features predictive for early neoplasia in Barrett's esophagus using high-quality histological correlation. Gastrointest Endosc 2017; 85: 918-926.e7

[29] Han J, Tsujino T, Samarasena JB et al. Tu1143 correlation of volumetric laser endomicroscopy (VLE with histology in patients with Barrett's esophagus and associated dysplasia: Does VLE add value to endoscopy? Gastrointest Endosc 2016; 83: AB551 - AB552

[30] Konda VJ, Navaneethan U, McKinley M et al. Sa1263 volumetric laser endomicroscopy identifies suspicious areas missed on white light endoscopy during surveillance of non-treated Barrett's esophagus. Gastroenterology 2016; 150: S261

[31] Leggett CL, Gorospe EC, Chan DK et al. Comparative diagnostic performance of volumetric laser endomicroscopy and confocal laser endomicroscopy in the detection of dysplasia associated with Barrett's esophagus. Gastrointest Endosc 2016; 83: 880 - 888.e2

[32] Swager AF, van der Sommen F, Klomp SR et al. Computer-aided detection of early Barrett's neoplasia using volumetric laser endomicroscopy. Gastrointest Endosc 2017; 86: 839-846

[33] Isenberg G, Sivak MV, Chak A et al. Accuracy of endoscopic optical coherence tomography in the detection of dysplasia in Barrett's esophagus: A prospective, double-blinded study. Gastrointest Endosc 2005; 62: 825-831

[34] Evans JA, Poneros JM, Bouma BE et al. Optical coherence tomography to identify intramucosal carcinoma and high-grade dysplasia in Barrett's esophagus. Clin Gastroenterol Hepatol 2006; 4: 38-43

[35] Chak A, Wallace MB, Poneros JM. Optical coherence tomography of Barrett's esophagus. Endoscopy 2005; 37: $587-590$

[36] Alshelleh M, Inamdar S, McKinley M et al. Incremental yield of dysplasia detection in Barrett's esophagus using volumetric laser endomicroscopy with and without laser marking compared with a standardized random biopsy protocol. Gastrointest Endosc 2018; 88: 35-42

[37] van der Sommen F, Klomp SR, Swager A-F et al. Predictive features for early cancer detection in barrett's esophagus using volumetric laser endomicroscopy. Comput Med Imaging Graph 2018; 67: 9-20 